\title{
PAIR ANNIHILATION EFFECTS IN MULTIQUARK SPECTROSCOPY
}

\author{
Chan Hong - Mo \\ CERN - Geneva \\ and \\ H. Högaasen \\ University of Oslo - Norway
}

\section{A B S T R A C T}

Pair annihilations in multiquark spectroscopy, in contrast to $Q \bar{Q}$ mesons, is an effect of leading order in $\alpha_{S}$ and cannot be consistently neglected. Their observation in experiment would help to confirm QCD as a quantum field theory, identify the quantum numbers of the gluon, and give information on possible states with gluon constituents. These effects are studied here theoretically in some detail in expectation of experimental data which are beginning to be available. 
1. To date, most of the success of quantum chromodynamics (QCD) in hadron spectroscopy has been obtained by exploiting its analogy ${ }^{1-3)}$ with quantum electrodynamics (QED) in atomic physics. For example, current models of charmonium are closely modelled on positronium. Thus, in place of the one-photon-exchange diagram (Fig. 1a) between $e^{+}$and $e^{-}$in positronium, which leads to the Fermi contact term

$$
H_{F}=-\frac{8 \pi}{3} \underline{\mu}_{1} \cdot \mu_{2} \delta^{3}\left(\underline{r}_{1}-\underline{r}_{2}\right), \quad \mu_{i}=\frac{e_{i} g_{i}}{2 \mu_{i}} \underline{s}_{i},
$$

we have the one-gluon-exchange diagram Fig. 1b between $\mathrm{c}$ and $\bar{c}$ in charmonium, which gives rise to the so-called colour magnetic interaction:

$$
H_{C M}=-\frac{8 \pi}{3} \sum_{a} \mu_{1}^{a} \mu_{2}^{a} \delta^{3}\left(Y_{1}-\underline{r}_{2}\right), \quad \underline{\mu}_{i}^{a}=\frac{\sqrt{\alpha_{s}}}{2 \mu_{i}} \frac{1}{2} \lambda_{i}^{a} \underline{s}_{i}
$$

and is believed to be responsible for the mass-splitting between $J / \psi$ and $\eta_{c}$, for example.

In positronium, however, there is another diagram of the same order in $\alpha$ which contributes to the mass difference between the ortho and para states, namely Fig. 2a, representing the virtual annihilation of $\mathrm{e}^{+} \mathrm{e}^{-}$into one photon, leading to the Pirenne annihilation potential ${ }^{4)}$ :

$$
H_{p}=\frac{2 \pi \alpha}{m_{1} m_{2}}\left\{\frac{3}{4}+\underline{s}_{1} \cdot \underline{s}_{2}\right\} \delta\left(\underline{r}_{1}-\underline{r}_{2}\right)
$$

whose effect is similax in magnitude to that of the Fermi contact term (1) and has clearly been identified in experiment. The existence of (3) is an unambiguous prediction -- and its observation therefore a triumph -- of the quantum field theoretical formulation of electrodynamics.

By analogy, there should be similar pair annihilation effects in QCD when applied to hadron spectroscopy. The only reason they are not considered in charmonium is that the annihilation diagram of leading order in $\alpha_{s}$, namely Fig. $2 b$, 
contributes only to $\mathrm{c} \overline{\mathrm{c}}$ pairs with colour 8. Annihilation effects in charmonium (or indeed in any $Q \bar{Q}$ meson) ${ }^{*}$ ) must therefore come from diagrams of order $\alpha_{s}^{2}$ or higher, which are arguably smaller (and in any case much harder to calculate).

There is no such argument, however, when considering a multiquark system in which a $Q \bar{Q}$ pair with colour 8 can occur. For example, take a $Q Q \bar{Q} \bar{Q}$ hadron, which though a total colour singlet can still be formed from two $Q \bar{Q}$ systems, each in a colour octet, thus: $\left[(Q \bar{Q})^{8}(Q \bar{Q})^{8}\right]^{l}$. For such system, therefore, it would be inconsistent not to take account of pair annihilation interactions which are of the same order in $\alpha_{s}$ as the colour magnetic interactions.

The object of this paper is to find situations where evidence for such firstorder annihilation effects can be obtained. Besides being necessary for consistency in multiquark spectroscopy, these effects are of general interest to particle physicists in that they exhibit

i) the quantum field theoretical aspects of the colour interaction between quarks and gluons which directly imply their existence;

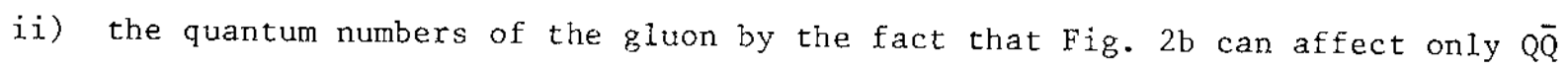
pairs carrying those quantum numbers, namely colour 8, flavour 0 , and total $J^{P}=1^{-}$.

We find two situations in which these pair annihilation effects may most easily be studied. The first is that of the swave QQQ̄Q hadrons, which have been considered by Jaffe in several papers ${ }^{5}{ }^{6}$, but in which annihilation effects have so far not been computed. Such effects will not only shift the masses in the spectrum but will also mix states with different quark contents, such as qqq̄ and qsiqs, thus affecting their decay. These hadrons, however, have the disadvantage of being extremely unstable (indeed, so unstable that they should not perhaps be regarded as hadron states at al1) and therefore experimentally elusive; also, there being two possible pairs of $\bar{Q}$ with colour 8 , each of which is affected by

*) In our notation, $Q$ may represent a quark of any flavour, while $q$ is reserved for $u$ or $d$. 
annihilation, the analysis is necessarily complex and indirect. The second situation is when the multiquark hadron is separated spatially into two portions by some angular momentum barrier, for example:

$$
(Q \bar{Q})^{x} \longrightarrow(Q \bar{Q})^{\bar{x}},(Q \bar{Q})^{x}-(Q Q Q)^{\bar{x}},(Q Q)^{x}-(Q Q \bar{Q})^{\bar{x}} \text {, etc. }
$$

in which case it has been argued that the hadron may be considered as a colour molecule constructed out of two colour ions ${ }^{7-13}$ ), each with its own properties amenable to individual investigation. Such molecules, if they exist, are much harder to produce experimentally than the s-wave QQQ̄Q hadrons discussed above, but once obtained and identified, may be an easier tool for studying effects of quark-pair annihilation. We shall study examples of both cases.

What form will the annihilation interaction take between $Q \bar{Q}$ pairs? If hadrons were indeed the analogues of positronium in which massive quarks interact perturbatively via the exchange of massless gluons, we may simply modify the Pirenne potential taking account of the colour factors, and obtain for the annihilation interaction between a quark and an antiquark

$$
H_{A N}=\frac{2 \pi \alpha_{s}}{m_{1} m_{2}}\left\{\frac{3}{4}+s_{1} \cdot \underline{s}_{2}\right\}\left\{\frac{1}{18}\left[\frac{16}{3}+\sum_{a} \lambda_{1}^{a} \lambda_{2}^{a}\right]\right\} \delta\left(\underline{r}_{1}-\underline{r}_{2}\right),
$$

where the second factor in (4) is just the colour 8 projection operator. In other words, besides the colour magnetic interaction between any quark pair $i$ and $j$ which we may unite as

$$
H_{1}=-\sum_{i j} c_{i j} \sum_{a}\left(\lambda_{i}^{a} \underline{\sigma}_{i}\right)\left(\lambda_{j}^{a} \underline{\sigma}_{j}\right)
$$

we have another term for annihilation:

$$
H_{2}=\sum_{i j} B_{i j} \cdot \frac{1}{4}\left\{3+\sigma_{i} \cdot \sigma_{j}\right\}\left\{\frac{1}{q}\left[\frac{16}{3}+\sum_{a} \lambda_{i}^{a} \lambda_{j}^{a}\right]\right\},
$$


where

$$
B_{i j}=6 C_{i j}
$$

It is understood in both (6) and (7) that when $i$ denotes an antiquark, we make the replacement: $\sigma_{i} \rightarrow-\sigma_{i}^{*}, \quad \lambda_{i} \rightarrow-\lambda_{i}^{*}$, while in (7) the sum in $i, j$ runs only over pairs of a quark and an antiquark of opposite flavours (gluons being by assumption flavourless).

Now, the values of the coefficients $C_{i j}$ in (6) can in many cases be estimated phenomenologically from the spectrum of ordinary $Q \bar{Q}$ mesons and QQQ baryons. If the analogy with positronium holds strictly, then we also know $B_{i j}$ from (8), and the spectrum of our s-wave multiquark systems can be calculated. Unfortunately, the successes of this analogy may be misleading and only work for the simplest systems such as charmonium. In the MIT bag ${ }^{2}$, for example, which in some respects is likely to give a more realistic picture for colour confinement, the light quarks $u$ and $d$ acquire their effective (constituent) masses of a few hundred $\mathrm{MeV}$ only via the confinement mechanism. In the same way, therefore, gluons will also acquire an effective mass when they are confined ${ }^{14}$. A modified perturbation theory will then have to be developed where the gluons emitted and absorbed exist in confined cavity modes with finite masses ${ }^{15,16}$ ). To a certain approximation, taking account only of the lowest gluon mode, the interactions (6) and (7) are still obtained for every pair of quark constituents, but now the relation (8) no longer holds. Depending on whether the gluon mode has a smaller or larger effective mass than the $Q \bar{Q}$ pair, the annihilation interaction is repulsive or attractive $\left(B_{i j}>0\right.$ or $\left.<0\right)$. Moreover, there can now be mixing between states with different numbers of gluons and of $Q \bar{Q}$ pairs. Calculations along these lines can be performed but will be strongly model-dependent. Unwilling, at present, to subscribe to any one specific confinement mechanism, we shall at first ignore the possible mixing between states with different numbers of $Q \bar{Q}$ pairs and gluons, and take $B_{i j}$ as free parameters to be determined phenomenologically. 
The values of $B_{i j}$ so determined will eventually give indirect information on the confining mechanism, in particular on the masses of hadron states with constituent gluons.

2. Having decided on the form of the interactions $H_{i}$, our next task is the calculation of their matrix elements. Examples for the evaluation of $\mathrm{H}_{1}$ matrices exist already in the literature. For these, therefore, we shall only give the results of our calculation which, although in some cases more laborious, is a not too devious generalization of earlier work, and concentrate instead on the less familiar $\mathrm{H}_{2}$. In contrast to the gluon-exchange interaction, the annihilation diagram mixes states with different quark contents. Nevertheless, because of their high masses, the mixing of states containing $c \bar{c}$ and $b \bar{b}$ pairs will not appreciably affect the properties of the lowest multiquark states which interest us. We shall therefore restrict our attention to $Q=u, d$, and $s$. For these, the experience gained with the colour magnetic interactions is that whereas it is quite feasible to neglect the differences between the light quarks $u$ and $d$, thereby keeping isospin symmetry, it is unrealistic, for practical purposes, not to account for the breaking in symetry between the flavours $q=u$, and $s^{8}$ ). Hence we shall do so also for the annihilation term $\mathrm{H}_{2}$.

We deal first with hadrons of the type (4) for which the calculation is technically simpler. We are primarily interested in narrow states which are experimentally easier to observe and identify. We shall therefore ignore those molecules formed from ions with colour charges $\mathrm{x}, \overline{\mathrm{x}}=1$ or 3 ; the first can decay simply by dissociation, and the second by rupturing the colour bond via the creation of a $\mathrm{q} \overline{\mathrm{q}}$ pair as in, for example, $f \rightarrow \pi \pi$, leading to widths typically much in excess of $100 \mathrm{MeV}^{9}$ ). We are then left, from formula (4), with the following molecules to consider:

$$
(Q \bar{Q})^{8}-(Q \bar{Q})^{8},(Q \bar{Q})^{8}-(Q Q Q)^{8},(Q Q)^{6}-(Q Q \bar{Q})^{\overline{6}},
$$

which contain two ions, $(Q \bar{Q})^{8}$ and $(Q Q \bar{Q})^{\overline{6}}$, affected by pair annihilations. 
For the simple $(Q \bar{Q})^{8}$ ions, only the spin triplet states $(Q \bar{Q})_{3}^{8}$ are affected by $\mathrm{H}_{2} ; \operatorname{explicitly~}$

$$
\begin{aligned}
& H_{2}(\mu \bar{\mu})_{3}^{8}=B_{0}(\mu \bar{\mu})_{3}^{8}+B_{0}(d d)_{3}^{8}+B_{1}(s \bar{s})_{3}^{8}, \\
& H_{2}(d \bar{d})_{3}^{8}=B_{0}(\mu \bar{\mu})_{3}^{8}+B_{0}(d d)_{3}^{8}+B_{1}(s \bar{s})_{3}^{8}, \\
& H_{2}(s \bar{s})_{3}^{8}=B_{1}(\mu \bar{\mu})_{3}^{8}+B_{1}(d \bar{d})_{3}^{8}+B_{2}(s \bar{s})_{3}^{8} .
\end{aligned}
$$

If we use as a basis the linear combinations of $(\mathrm{q} \overline{\mathrm{q}})_{3}^{8}$ states which have definite isospins,

$$
\begin{array}{lll}
(q \bar{q})_{3,3}^{8}=\frac{1}{\sqrt{2}}(\mu \bar{\mu}-d d)_{3}^{8} & I=1, \\
(q \bar{q})_{3,1}^{8}=\frac{1}{\sqrt{2}}(\mu \bar{\mu}+d \bar{l})_{3}^{8} & I=0,
\end{array}
$$

we have instead

$$
\begin{aligned}
& H_{2}(q \bar{q})_{3}^{8}=0 \quad I=1 \\
& H_{2}(q \bar{q})_{3}^{8}=2 B_{0}(q \bar{q})_{3}^{8}+\sqrt{2} B_{1}(5 \bar{s})_{3}^{8} \\
& \left.H_{2}(s \bar{s})_{3}^{8}=\sqrt{2} B_{1}(q \bar{q})_{3}^{\gamma}+B_{2}(s \bar{s})_{3}^{8}\right\} I=0
\end{aligned}
$$

with no effect on the $I=1$ channel, thus breaking the 'exchange' degeneracy between $I=1$ and 0 in the $u$, $a$ sector, while in $I=0$, it mixes the state ( $q \bar{q})$ with the $(\mathrm{s} \overline{\mathrm{s}})$. From (11), the matrix elements of $\mathrm{H}_{2}$ can immediately be read off.

For the ions $(Q Q \bar{Q})^{6}$ the calculation is more complicated, there being now two terms in $\mathrm{H}_{2}$ corresponding to the two $\bar{Q}$ pairs in the ion. Fortunately, since total colour equals 6 implies that both these $Q \bar{Q}$ pairs must have colour 8 , we may omit altogether from (7) the second factor with $\lambda$ matrices corresponding to the colour 8 projection operator, leaving only Pauli matrices which one can easily handle. The diagonalization of isospin in the $u$, d sector becomes more involved also, but it is no different in principle from that of the $(Q \bar{Q})^{8}$ ions discussed above. 
The matrix elements of $\mathrm{H}_{1}$ and $\mathrm{H}_{2}$ so calculated for all the ions in (4) are listed together in Tables 1 to $4^{*}$ ).

Next, we consider the $(Q Q \bar{Q} \bar{Q})$ hadrons in which all the quark constituents are in the s-wave. There being now an enormous number of states, we shall not at present consider all of them. We shall first cut the problem approximately in half by restricting our attention to only the $\mathrm{J}^{\mathrm{P}}=0^{+}$and $2^{+}$sector for which some possible experimental candidates exist. We shall use as the basis the states where the diquark $(Q Q)$ (antidiquark $\bar{Q} \bar{Q}$ ) has colour representation $x(\bar{x})$ and spin multiplicity $S(S)$, A state will be written $\left[(Q Q){ }_{S}^{x}(\bar{Q} \bar{Q}){ }_{S}^{\bar{x}}\right]_{+}$if $Q Q$ and $\overline{Q Q}$ are symmetric in flavour, and $\left[(Q Q)_{S}^{\mathrm{X}}(\bar{Q} \bar{Q})_{S--}^{\bar{x}}\right]$ if they are antisymmetric in flavour. This basis has the virtue that the colour-magnetic Hamiltonian $H_{1}$ is almost diagonal. The annihilation Hamiltonian $\mathrm{H}_{2}$ is now a sum of four terms corresponding to the four combinations of $Q \bar{Q}$ pairs, say $1 \overline{1}, 2 \overline{2}, 1 \overline{2}$, and $2 \overline{1}$. In order to calculate the matrix element of the term $1 \bar{l}$, we can first express the state $\left[(O Q)_{S}(\vec{Q} \bar{Q}){ }_{S}^{\bar{x}}\right]$ in terms of the states $\left[\left(Q_{1} \bar{Q}_{2}\right)_{S^{\prime}}^{x^{\prime}}\left(Q_{2} \bar{Q}_{2}\right)_{S^{\prime}} \bar{x}^{\prime}\right]$ with $x^{\prime}=1$ or 8 and $S^{\prime}=1$ or 3 , via colour and spin crossing matrices. Only the state with $\mathrm{x}^{\prime}=8$ and $\mathrm{S}^{\prime}=3$ gives a non-vanishing matrix element for the term $1 \overline{1}$, namely $B_{1}$. The other terms of $\mathrm{H}_{2}$ can be similarly computed. Also, analogously to (11), we can diagonalize the u, d sector by choosing, as the basis, total isospin eigenstates. The final results are 1 isted in Tables 5,6 and 7.

3. Although several candidates already exist in the experimental data for the multiquark states of interest to us here, the information available is as yet insufficient for their unambiguous assignment to the complex spectrum. We must therefore still rely on the spectroscopy of ordinary mesons and baryons for the phenomenological determination of our parameters. From an analysis of baryon states, we can estimate quite reliably the values of the parameters $C$ in (6) between $Q Q$ pairs $\left.{ }^{7}, 8,10\right)$. We shall use

*) The contents of Table 1 exist already in the literature ${ }^{9}$. They are 1 isted here again for completeness. 


$$
C_{0}=20 \mathrm{MeV}, \quad C_{1}=12.5 \mathrm{MeV}, \quad C_{2}=9.0 \mathrm{MeV},
$$

and a mass difference between $s$ and $u$, d quarks

$$
\Delta M=175 \mathrm{MeV} \text {, }
$$

which represents a sort of consensus of several fits. A parallel analysis of meson states gives usually somewhat larger estimates for $\mathrm{C}$ between $Q \bar{Q}$ pairs, but we are not yet in a position to worry about such differences and shall ignore them at present. From our previous discussion it is obvious that ordinary meson and baryon spectroscopy cannot yield direct information on the annihilation parameters $B$ in (7). We cannot thus as yet attempt to calculate the spectrum of states or make explicit predictions for direct comparison with experiment. Nevertheless, we shall show that it is possible to have a good idea of what can be learnt when the data become available.

Consider first the simple ion $(Q \bar{Q})_{3}^{8}$. If we believe the description in terms of massive constituent quarks interacting via a potential due to gluon exchange, then the relation (8) holds and the spectrum can be calculated. The annihilation potential is repulsive, pushing up the masses of the $I=0$ states, and mixes the $(q \bar{q})$ and $(s \bar{s})$ components. From (13) and (14) we obtain for the two $I=0$ eigenstates, say $\omega^{8}$ and $\phi^{8}$, mass differences of the order of $190 \mathrm{MeV}$ and $460 \mathrm{MeV}$, respectively, above the unaffected $I=1$ state $\rho^{8}$, and a mixing angle between $q \bar{q}$ and $\bar{s} \bar{s}$ of the order of $\tan \theta \sim 0.45$. Such large mass differences can, of course, be easily detected, while the large mixing angle means a complete break-down of the OZI rule, so that $\phi^{8}$ becomes unstable, decaying readily into $\rho^{8}+\pi$. If, on the other hand, the MIT bag picture is correct and B is much smaller than what (8) requires or even attractive, it will be reflected in the mass shifts, while the OZI rule will be better preserved. Experimental observation of these mass shifts (relative to $\rho^{8}$ and $\eta^{8}$, say) and mixings, when ascribed to first-order annihilation effects, means that the gluon must have colour 8 , isospin 0 , and spin-parity $1^{-}$as expected, while the direction of the mass shift and the size of the mixing 
will tell us the sign of $B$, and hence whether the ion with a single gluon as constituent is lighter or heavier than the $(\bar{q} \bar{q})^{8}$ ion.

Similar considerations can, of course, be applied to the ions $(Q Q \bar{Q})^{\overline{6}}$. The distinction of annihilation effects between states with different quantum numbers will not be as clear as for $(Q \bar{Q})^{8}$; nevertheless, the fact that the gluon carries flavour 0 and spin 1 means that states with low flavour and high spin are in general more strongly affected. Again the potential picture implies positive mass shifts and large OZI violations, making some otherwise stable states unstable, as indicated in Table 4 -- whereas for the bag picture of confinement, the situation would be quite different.

Of course $(Q \bar{Q})^{8}$ cannot exist except in combination with another ion of the opposite colour charge, say $(Q \bar{Q})^{8}$ or $(Q Q Q)^{8}$. There is as yet no candidate for $(Q \bar{Q})^{8}-(Q \bar{Q})^{8}$ in experiment, but several possibilities are known for $(Q \bar{Q})^{8}-(Q Q Q)^{8}$ baryons. In particular, three states with masses $2.13,2.26$, and $2.58 \mathrm{GeV} 17-19$ ) decaying into $\Lambda \pi^{+} \pi^{-}, \Sigma^{-} \omega+\Lambda \pi^{+} \pi^{-} \pi^{-}$, and $\mathrm{K}^{+} \mathrm{K}_{\mathrm{S}^{0}} \Sigma^{-}$, respectively, were reported; these have the correct quantum numbers to be assigned to $\omega^{8}$-(uds) ${ }^{8}, \rho^{8}$-(uds) ${ }^{8}$, and $\phi^{8}-(\text { uds })^{8}$, respectively, where the $\left(\right.$ uds) ${ }^{8}$ ion could be the (qqs) ${ }_{2}^{8}$ ion with $I=0$ in Table 3 which has an exceptionally large colour-magnetic attraction and therefore the lowest mass. If so, the negative mass shift between $\omega^{8}$ and $\rho^{8}$, plus the apparent validity of the OZI rule in the decay of (2.58) into $\mathrm{K}^{+} \mathrm{K}_{\mathrm{S}}^{0} \Sigma^{-}$, would suggest that the annihilation interaction is attractive and that a constituent gluon is heavier than a q $\bar{q}$ pair. The parameters thus obtained are:

$$
B_{0} \sim-60 \mathrm{MeV}, B_{2} \sim-30 \mathrm{MeV}, B_{1} \sim-\sqrt{B_{0} B_{2}} \text {, }
$$

with a mixing angle $\theta$ between $(\mathrm{q} \overline{\mathrm{q}})^{8}$ and $\left(\mathrm{s}_{\overline{\mathrm{s}}}\right)^{8}$ of about $10^{\circ}$.

Unfortunately, both the existence and the assignment of these states are at present very tentative. The assignment of baryons to QQQQQ states is particularly tricky since there are two families of metastable molecules in approximately the same mass range, namely $(Q \bar{Q})^{8}-(Q Q Q)^{8}$ and $(Q Q)^{6}-(Q Q \bar{Q})^{6}$. However, depending on whether the annihilation interaction is repulsive or attractive, the expected 
properties of the molecules are quite different, thus substantially affecting their possible assignments to observed resonances. Take, for example, the state (2.58) quoted above, seen decaying into $\mathrm{K}^{+} \mathrm{K}_{\mathrm{S}}^{0} \Sigma^{-}$, or another recently reported ${ }^{20}$ ) state at $3.17 \mathrm{GeV}$, also with multistrange decay modes. They have hypercharge zero, but instead of giving decay products containing only one strange particle as is usual for $S=-1$ resonances, they prefer final states with $\Sigma|S|=3$. This presumably implies that they contain two $s$ and one $\bar{s}$ constituent quarks and are therefore at least quss $\bar{s}$ systems. In our picture of these states as chromionic molecules there are five possibilities for the colour and flavour separations:

$$
\begin{aligned}
& (q \bar{s})^{8}-(q s s)^{8},(s \bar{s})^{8}-(q q s)^{8} ; \\
& (s s)^{6}-(q q \bar{s})^{6},(q s)^{6}-(q s \bar{s})^{6},(q q)^{6}-(s s \bar{s})^{\overline{6}}
\end{aligned}
$$

in addition to various possibilities for the ionic spins. However, if annihilation is strongly repulsive as in (8), many of the ions in (16) become unstable by OZI violations (see, for example, Table 4), thus excluding some of the possible assignments. In particular, the ion $\left(\mathrm{s}_{\bar{s}}\right)_{3}^{8}$ becomes maximally mixed with $(\mathrm{q} \overline{\mathrm{q}})_{3}^{8}$, so that the tentative assignment in the preceding paragraph of $(s \bar{s})_{3}^{8}-(\text { uds })_{2}^{8}$ to the (2.58) state would be untenable although $(s \bar{s})_{1}^{8}-\left(u_{d s}\right)_{2}^{8}$ is still possible.

Let us now turn to the s-wave QQQQQQ hadron states. Jaffe has argued ${ }^{5,6)}$ quite viably that the $0^{+}$structures seen in low-energy meson-meson scattering should be associated with these $Q Q \bar{Q} \bar{Q}$ s-wave states rather than with the p-wave excitations of $Q \bar{Q}$ expected in about the same mass range. Moreover, quite recently there are reports in the $2 \mathrm{GeV}$ region of $2^{+}$resonances from experiments associated with nucleon-antinucleon channels ${ }^{21}$ ) which are arguably therefore also candidates for

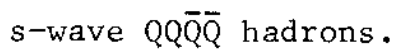

One difficulty with these states, however, is the fact that we know of no mechanism to prevent their constituents from combining into two colour-singlet $Q \bar{Q}$ pairs. There being then no colour forces to hold these neutral objects together, the system can simply dissociate into two mesons, in contradiction to what is normally expected from a genuine hadron state. In view of this, Jaffe and Low 
suggested in a recent paper ${ }^{22}$ ) that these states be considered not as resonances but only as poles of the P-matrix (i.e. the inversed Wigner-Eisenbud R-matrix) which they call 'primitives'. With such an interpretation, they were able to obtain from the s-wave (QQDQD) bag states a reasonable description of low-energy meson-meson scattering data. In particular, the $S^{*}(980)$ and $\delta(980)$ mesons with $I^{G}\left(J^{P}\right)=0^{+}\left(0^{+}\right)$and $1^{-}\left(0^{+}\right)$, respectively, were both ascribed to the lowest primitives with qsqus constituents. However, there is a difficulty with these assignments in that a pure qs $\bar{q} \bar{s}$ state can decay only into $k \bar{K}$ by dissociation, not into $\pi \pi$ or, to the same extent, $\pi n$, whereas experimentally both the latter modes appear

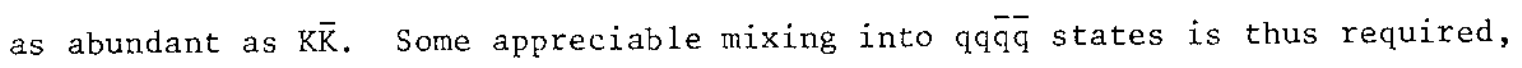
but this cannot arise from the colour-magnetic interaction to which their calculation was restricted.

Now, the annihilation mechanism we are considering would lead naturally to

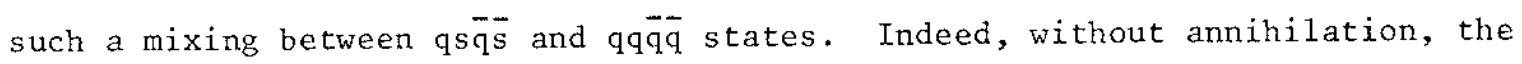
lowest qsqs state is antisymetric in flavour -- in our notation (qs $\bar{q} \bar{s}$ ) which lies much higher in mass than the corresponding ( $\mathrm{qq} \overline{\mathrm{q}} \overline{\mathrm{q}}$ ) - states because of the

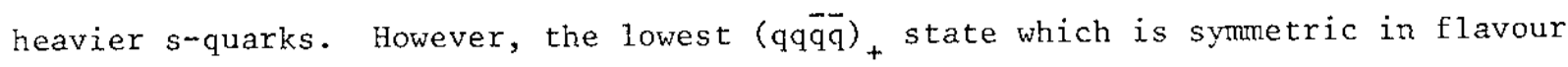

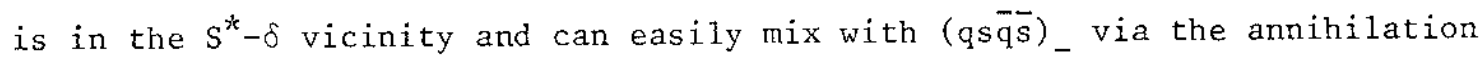
interactions. Indeed, by diagonalizing the appropriate matrices in Table 5, one finds that maximal mixing between these states in both $\mathrm{I}^{G}=0^{+}$and $\mathrm{I}^{-}$is obtained for an attractive annihilation with $\mathrm{B} \sim-60 \mathrm{MeV}$ [intriguingly similar to the value quoted in (16)] without disturbing the other positive features of the Jaffe-Low spectrum, such as the near-degeneracy between $S^{*}$ and $\delta$. of course, this description is far from definitive, since it is not excluded that one can obtain equally good 'fits' with different choices of parameters, but it is at least an interesting possibility, although the theoretically predicted residue is still too smal1 ${ }^{23)}$.

One trouble with the $0^{+}$mesons is their great complexity, witness the size of the matrices in Table 5 not to mention the possible mixing also with $(Q \bar{Q})$ and $(Q \bar{Q} g)$ states ${ }^{24)}$. It may be more profitable to select for study a less populated channel in the $(Q Q \bar{Q} \hat{n})$ spectrum, such as the $\mathrm{J}^{\mathrm{P}}=2^{+}$states. Some attempts in that 
direction were made, but no definite conclusion can yet be extracted from the existing data.

In summary, it would appear that with the Tables 1 to 7 , both the detailed phenomenological analysis of annihilation effects and the calculation of the hadronic spectxa will be a feasible enterprise once better data become available.

\section{Acknowledgements}

Much of this investigation was performed when one of us (C.H.M.) was at the Rutherford Laboratory. Some assistance in calculation from T.H. Hansson is gratefully acknowledged. 


\section{REFERENCES}

1) A. de Rújula, H. Georgi and S.L. Glashow, Phys. Rev. D 12 (1975) 147.

2) T. De Grand et al., Phys. Rev. D 12 (1975) 2060.

3) See, for example, A.J.G. Hey, Proc. EPS Int. Conf. on High-Energy Physics, Geneva, 1979 (CERN, Geneva, 1980), Vo1. 2, p. 523.

4) J. Pirenne, Arch. Sci. Phys. Nat. 29 (1947) 207.

5) R.L. Jaffe, Phys. Rev. D $\underline{15}$ (1977) 267 and 281.

6) R.L. Jaffe, Phys. Rev. D 17 (1978) 1444.

7) Chan Hong-Mo and H. Högaasen, Phys. Lett. $\underline{72 B}$ (1977) 121; Nuc1. Phys. B136 (1978) 401.

8) Tsou Sheun Tsun, Nuc1. Phys. B141 (1978) 397.

9) Chan Hong-Mo et a1., Phys. Lett. 76B (1978) 634.

10) H. Högaasen and P. Sorba, Nucl. Phys. B145 (1978) 119.

11) M. De Crombrugghe, H. Högaasen and P. Sorba, Nucl. Phys. B156 (1979) 347.

12) M. Fukugita, K. Konishi and T.H. Hansson, Phys. Lett. 74B (1978) 261.

13) D.M. Tow, B. Nicolescu and J.M. Richard, Nuc1. Phys. B150 (1979) 287.

14) A finite effective gluon mass has also been suggested in a recent jet analysis, G. Parisi and R. Petronzio, CERN preprint TH 2804 (1980).

15) T.D. Lee, Phys. Rev. D 19 (1979) 1802.

16) F.E. Close and R.R. Horgan, Rutherford Laboratory preprint RL-79-067 (1979).

17) W. Lockman et a1., Saclay preprint D.Ph.PE 78-01 (1978).

18) W. Lockman et a1., UCLA preprint 1109 (1978). Amsterdam-CERN-Ni jmegen-Oxform Collaboration, submitted paper cited by R.A. Salmeron in Proc. European Conf. on Particle Physics, Budapest, 1977 (eds. L. Jenik and I. Montvay) (Central Research Institute for Physics, Budapest 1978), p. 565.

19) Amsterdam-CERN-Nijmegen-Oxford Collaboration, paper submitted to the 19 th Int. Conf. on High-Energy Physics, Tokyo, 1978; CERN preprint EP/Phys. 78-40 (1978).

20) Birmingham-CERN-Glasgow-Michigan State-Paris LPNHE Collaboration, J. Amirzadeh et al., Phys. Lett. 89B (1979) 125.

21) C. Defoix et a1., Collège de France preprint LPC/79-13 (1979).

22) R.I. Jaffe and F.E. Low, Phys. Rev. D 19 (1979) 2105.

23) H. Högaasen, Proc. of the Workshop on Baryonium and other Unusual Hadron States, Orsay, 1979 (eds. B. Nicolescu, J.M. Richard and R. Vinh Mau) (Orsay, 1979).

24) D. Horn and J. Mandula, Phys. Rev. D 17 (1978) 898.

T. Barnes, Nuc1. Phys. B152 (1979) $17 \overline{1}$. 
Table 1

\begin{tabular}{|c|c|c|}
\hline Ions & I & $\left\langle\mathrm{H}_{1}\right\rangle$ \\
\hline$(\mathrm{qq})_{3}^{6}$ & 0 & $-\frac{4}{3} \mathrm{C}_{0}$ \\
\hline$(\mathrm{qq})_{1}^{6}$ & 1 & $4 \mathrm{C}_{0}$ \\
\hline$(\mathrm{qs})_{3}^{6}$ & $\frac{1}{2}$ & $-\frac{4}{3} \mathrm{C}_{1}$ \\
\hline$(\mathrm{qs})_{1}^{6}$ & $\frac{1}{2}$ & $4 \mathrm{C}_{1}$ \\
\hline$(\mathrm{ss})_{1}^{6}$ & 0 & $4 \mathrm{C}_{2}$ \\
\hline
\end{tabular}

Table 2

\begin{tabular}{|c|c|c|c|}
\hline Ions & I & $\left\langle\mathrm{H}_{1}\right\rangle$ & $\left\langle\mathrm{H}_{2}\right.$ \\
\hline$(\mathrm{q} \overline{\mathrm{q}})_{3}^{8}$ & 0 & $-\frac{2}{3} \mathrm{C}_{0}$ & \\
\hline$(s \bar{s})_{3}^{8}$ & 0 & $-\frac{2}{3} C_{2}$ & $\sqrt{2} B_{1}$ \\
\hline$(q \bar{q})_{3}^{8}$ & 1 & $-\frac{2}{3} \mathrm{C}_{0}$ & 0 \\
\hline$(q \bar{q})_{1}^{8}$ & 0 & $2 \mathrm{C}_{0}$ & 0 \\
\hline$(\mathrm{q} \overline{\mathrm{q}})_{1}^{8}$ & 1 & $2 \mathrm{C}_{0}$ & 0 \\
\hline$(\mathrm{q} \overline{\mathrm{s}})_{3}^{8}$ & $\frac{1}{2}$ & $-\frac{2}{3} C_{1}$ & 0 \\
\hline$(\mathrm{q} \overline{\mathrm{s}})_{\mathrm{I}}^{8}$ & $\frac{1}{2}$ & $2 C_{1}$ & 0 \\
\hline$(s \bar{s})_{1}^{8}$ & 0 & $2 \mathrm{C}_{2}$ & 0 \\
\hline
\end{tabular}


Table 3

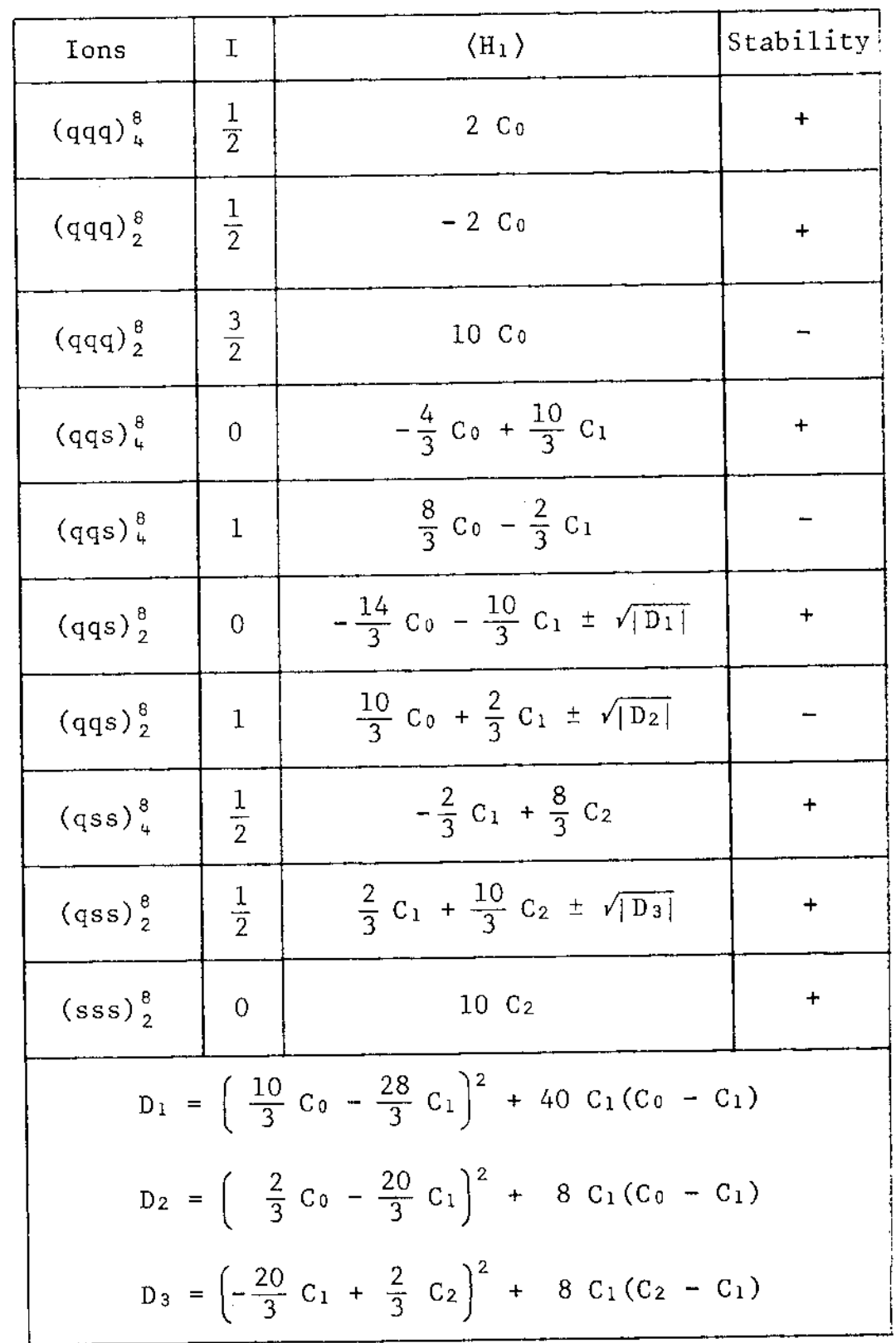


Table 4

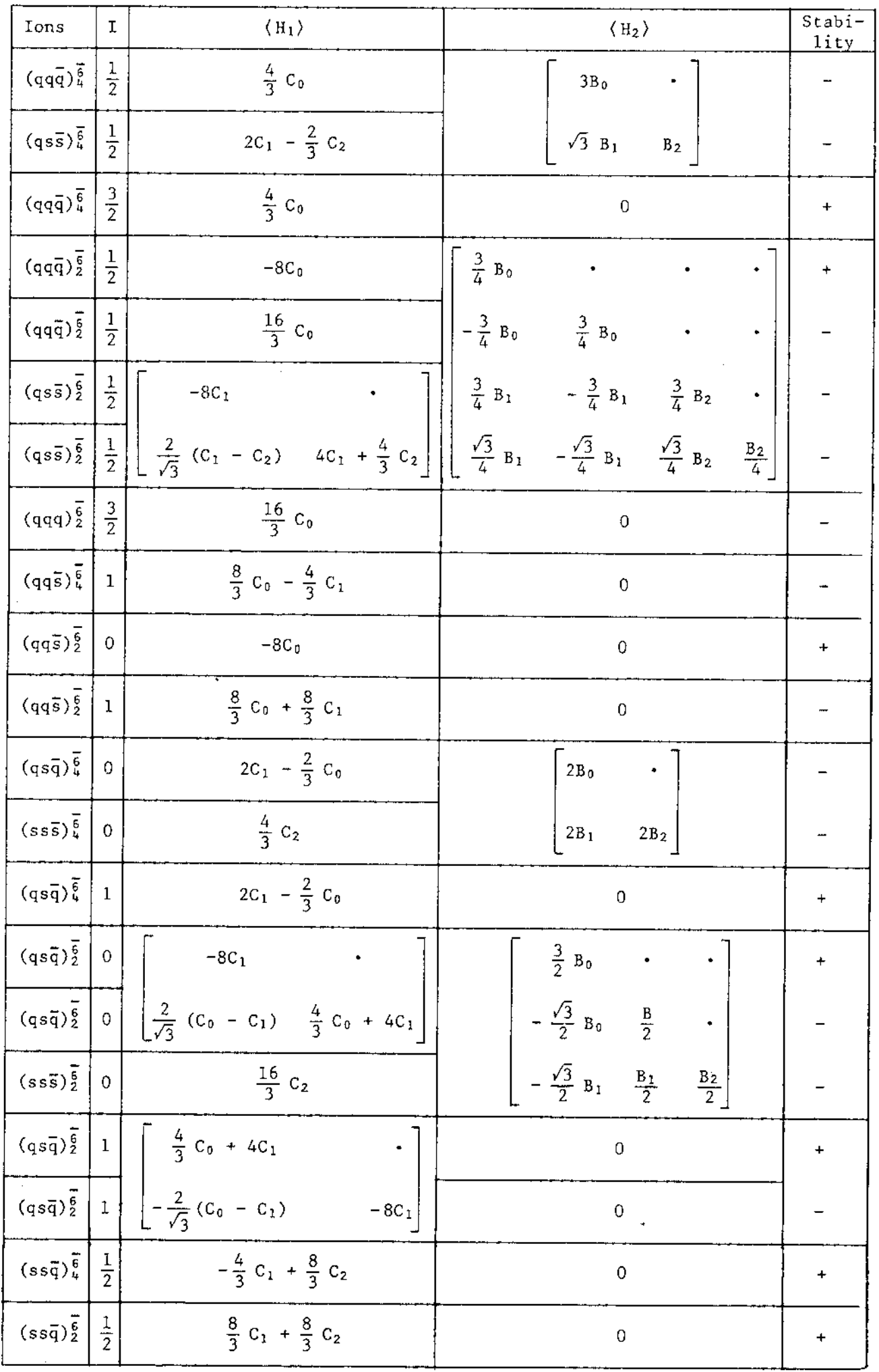


Table 5

\begin{tabular}{|c|c|c|c|c|}
\hline$I^{G}\left(J^{P}\right)$ & State & \multicolumn{2}{|l|}{$\left\langle\mathrm{H}_{1}\right\rangle$} & $\left\langle\mathrm{H}_{2}\right\rangle$ \\
\hline \multirow[t]{5}{*}{$0^{+}\left(0^{+}\right)$} & {$\left[(\mathrm{qq})_{1}^{\overline{3}}(\overline{\mathrm{q}} \overline{\mathrm{q}}) \mathrm{l}_{1}^{3}\right]$.} & $-16 \mathrm{C}_{0}$ & & \multirow{10}{*}{ ANNEX A] } \\
\hline & {$\left[(q \mathrm{q})_{3}^{6}(\overline{\mathrm{q}} \overline{\mathrm{q}})_{3}^{\overline{6}}\right]_{-}$} & $-8 \sqrt{6} c_{0}$ & $\frac{88}{3} c_{0}$ & \\
\hline & {$\left[(\mathrm{qs})_{1}^{\overline{3}}(\overline{\mathrm{q}} \overline{\mathrm{s}}){ }_{1}^{3}\right]$} & $-16 C_{1}$ & $\cdot$ & \\
\hline & {$\left[(\mathrm{qs})_{3}^{6}(\overline{\mathrm{q}} \mathrm{s})_{3}^{\overline{6}}\right]_{-}$} & $-2 \sqrt{6}\left(C_{0}+2 C_{1}+C_{2}\right)$ & $\left.\frac{4}{3}\left(5 C_{0}+12 C_{1}+5 C_{2}\right)\right]$ & \\
\hline & {$\left[(q q){ }_{3}^{\overline{3}}(\bar{q} \bar{q})_{3}^{3}\right]_{+}$} & & & \\
\hline \multirow{5}{*}[\begin{array}{c}{\text{see}}\\
{\text{footnote}}\end{array}]{} & {$\left[(\mathrm{qq})_{1}^{6}(\overline{\mathrm{qq}} \overline{\mathrm{q}})_{1}^{\overline{6}}\right]_{+}$} & $-8 \sqrt{6} C_{0}$ & $8 \mathrm{C}_{0}$ & \\
\hline & {$\left[(q s)_{3}^{\overline{3}}(\bar{q} \bar{s})_{3}^{3}\right]_{+}$} & {$\left[-\frac{8}{3}<C_{0}+\right.$} & $\cdot]$ & \\
\hline & {$\left[(q s){ }_{1}^{6}(\bar{q} \bar{s})_{1}^{\overline{6}}\right]_{+}$} & $-2 \sqrt{6}\left(c_{0}+2 c_{1}\right.$ & $\left.\left.+C_{2}\right) \quad 8 C_{1}\right]$ & \\
\hline & {$\left[(s s)_{3}^{\overline{3}}(\bar{s} \bar{s})_{3}^{3}\right]_{+}$} & {$\left[-\frac{16}{3} c_{2}\right.$} & & \\
\hline & {$\left[(s s)_{1}^{6}(\bar{s} \bar{s})_{1}^{\overline{6}}\right]_{+}$} & $-8 \sqrt{6} C_{2}$ & & \\
\hline \multirow[t]{6}{*}{$1^{-}\left(0^{+}\right)$} & {$\left[(\mathrm{qs}){ }_{1}^{\overline{3}}(\overline{\mathrm{q}} \mathrm{s}){ }_{1}^{3}\right]_{-}$} & $-16 \mathrm{C}_{1}$ & $\cdot$ & \multirow{6}{*}[\begin{array}{lll}{\text{ANNEX}}&{B}\end{array}]{} \\
\hline & {$\left[(\mathrm{qs}){ }_{3}^{6}(\overline{\mathrm{q}} \overline{\mathrm{s}})_{3}^{\overline{6}}\right]$} & $-2 \sqrt{6}\left(C_{0}+2 C_{1}+C_{2}\right)$ & $-\frac{4}{3}\left(5 C_{0}+12 C_{1}+5 C_{2}\right)$ & \\
\hline & {$\left[(\mathrm{qq})_{3}^{\overline{3}}(\overline{\mathrm{q}} \overline{\mathrm{q}})_{3}^{3}\right]_{+}$} & $-\frac{16}{3} c_{0}$ & & \\
\hline & {$\left[(\mathrm{qq})_{1}^{6}(\overline{\mathrm{q}} \overline{\mathrm{q}}) \overline{\mathrm{i}}\right]_{+}$} & $-8 \sqrt{6} C_{0}$ & $8 \mathrm{C}_{0}$ & \\
\hline & {$\left[(\mathrm{qs}){ }_{3}^{-\overline{3}}(\overrightarrow{\mathrm{q}} \overline{\mathrm{s}}){ }_{3}^{3}\right]_{+}$} & {$\left[-\frac{8}{3}\left(C_{0}+\right.\right.$} & 2) & \\
\hline & {$\left[(\mathrm{qs})_{1}^{5}(\overline{\mathrm{q}} \overline{\mathrm{s}})_{1}^{\overline{6}}\right]_{+}$} & $-2 \sqrt{6}\left(c_{0}+2 C\right.$ & $\left.\left.+C_{2}\right) \quad 8 C_{1}\right]$ & \\
\hline \multirow[t]{2}{*}{$2^{+}\left(0^{+}\right)$} & $\left.[(\mathrm{qQ}))_{3}^{\overline{3}}(\overline{\mathrm{q}} \overline{\mathrm{q}})_{3}^{3}\right]_{+}$ & $-\frac{16}{3} C_{0}$ & & 0 \\
\hline & {$\left[(\mathrm{qq})^{6}\left(\overline{\mathrm{q}}(\overline{\mathrm{q}} \overline{\mathrm{q}})^{\overline{6}}{ }_{+}\right]\right.$} & $-8 \sqrt{6} C_{0}$ & $\left.8 \mathrm{C}_{0}\right]$ & 0 \\
\hline \multicolumn{5}{|c|}{$\begin{array}{l}\text { In the } \mathrm{I}^{G}\left(\mathrm{~N}^{\mathrm{P}}\right)=\mathrm{O}^{+}\left(0^{+}\right) \text {sector }\left\langle\mathrm{H}_{1}\right\rangle \text { has in addition the following small off-diagonal } \\
\text { elements: }\end{array}$} \\
\hline & $\left\langle\mathrm{H}_{1}\right\rangle$ & {$\left[(\mathrm{qs})_{1}^{\overline{3}}(\overline{\mathrm{q}} \mathrm{s}){ }_{1}^{3}\right]$} & {$\left[(\mathrm{qs})_{3}^{6}(\overline{\mathrm{q} s})_{3}^{\overline{6}}\right]$} & \\
\hline & {$\left[(\mathrm{qs})_{3}^{\overline{3}}(\overline{\mathrm{q}} \overline{\mathrm{s}}){ }^{\frac{3}{3}}\right]+$} & $-\frac{4}{\sqrt{3}}\left(C_{0}-2 C_{1}+C_{2}\right)$ & $-4 \sqrt{2}\left(C_{0}-2 C_{1}+C_{2}\right)$ & \\
\hline & {$\left[(q \mathrm{q}){ }_{1}^{6}(\overline{\mathrm{q}} \overline{\mathrm{s}})_{\mathrm{1}}^{\overline{6}}\right]_{+}$} & 0 & $-\frac{10}{\sqrt{3}}\left(C_{0}-2 C_{1}+C_{2}\right)$ & \\
\hline
\end{tabular}


$-18-$

\begin{tabular}{|c|c|c|c|c|c|c|c|c|c|}
\hline - & • & - & - & • & • & - & • & . & $\mathscr{\infty}^{\circ}$ \\
\hline • & • & • & - & - & - & - & - & $\begin{array}{c}N \\
\infty \\
N / m\end{array}$ & Nim \\
\hline - & $\cdot$ & • & - & - & • & - & $\begin{array}{c}\overbrace{\infty}^{N} \\
-10 \\
+ \\
\infty \\
-1 N\end{array}$ & $\begin{array}{c}\vec{A} \\
-110\end{array}$ & $\begin{array}{c}\vec{\infty} \\
-\mid N\end{array}$ \\
\hline - & • & • & - & • & - & 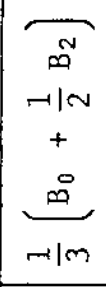 & $\begin{array}{c}\overbrace{\infty}^{N} \\
-1 / N \\
+ \\
\infty \\
-1 / \infty\end{array}$ & $\begin{array}{c}\vec{m} \\
N / m\end{array}$ & $\begin{array}{c}\vec{n} \\
-\mid N S\end{array}$ \\
\hline - & - & - & - & - & $\stackrel{\circ}{\infty}$ & $\begin{array}{c}-\vec{a} \\
-1 N\end{array}$ & $\underbrace{\vec{m}}$ & 0 & 0 \\
\hline • & • & • & • & م. & $\underbrace{\infty}$ & $\mid \begin{array}{c}m \\
-15\end{array}$ & $\vec{m}$ & 0 & 0 \\
\hline - & - & - & $\begin{array}{c}\overbrace{\infty}^{N} \\
-10 \\
+ \\
\infty \\
\infty 16\end{array}$ & $-\vec{\infty}$ & $-\left.\right|_{1} ^{\vec{m}}$ & $\begin{array}{c}\overbrace{m}^{N} \\
-\mid N \\
+ \\
\underbrace{\infty}_{1} \\
\left.\right|_{1} ^{\infty}\end{array}$ & 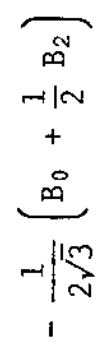 & $\begin{array}{c}\infty \\
-1 / m \\
1\end{array}$ & $\begin{array}{c}\vec{m} \\
-1 / \infty \\
1\end{array}$ \\
\hline - & - & $\begin{array}{c}\infty \\
\infty \\
+\mid N \\
+ \\
\infty\end{array}$ & $\begin{array}{c}\overbrace{\infty}^{N} \\
-1 N \\
+ \\
\infty \\
-15 \\
-15\end{array}$ & $-\overrightarrow{0}$ & $\begin{array}{c}\vec{n} \\
\operatorname{mon} \\
1\end{array}$ & $\begin{array}{c}\overbrace{\infty}^{N} \\
-1 / \infty \\
+ \\
\infty \\
-1 \mid \stackrel{m}{\infty}\end{array}$ & 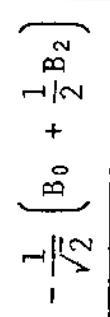 & $\underbrace{m}_{1}$ & $\begin{array}{c}\overrightarrow{D Q} \\
1\end{array}$ \\
\hline • & $\begin{array}{c}09 \\
-1 / 0\end{array}$ & $\begin{array}{c}\vec{m} \\
-\| N\end{array}$ & 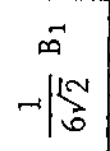 & $\begin{array}{c}\infty \\
-140 \\
1\end{array}$ & $\prod_{1}^{\infty}$ & $\begin{array}{l}\overrightarrow{9} \\
\rightarrow 10\end{array}$ & 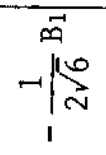 & 0 & 0 \\
\hline ஜ & $\begin{array}{c}\text { 保 } \\
-1 \mid\end{array}$ & $\begin{array}{c}\vec{\infty} \\
-115\end{array}$ & 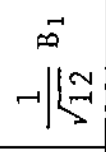 & $\stackrel{\circ}{\infty}$ & $\underbrace{\infty}_{1}$ & $\begin{array}{c}\vec{n} \\
-\mid \\
-\mid\end{array}$ & $\begin{array}{c}-\overrightarrow{0} \\
-1 N \\
1\end{array}$ & 0 & 0 \\
\hline 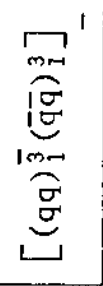 & 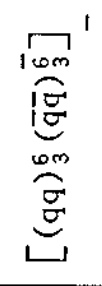 & 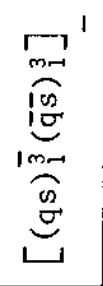 & 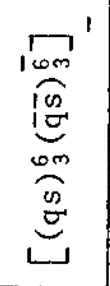 & 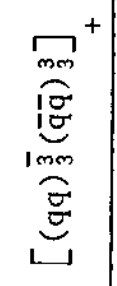 & 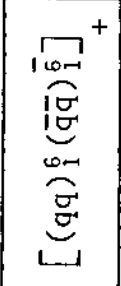 & 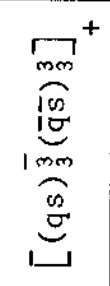 & 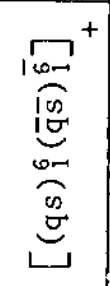 & 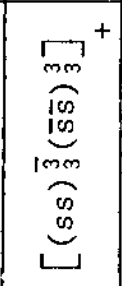 & 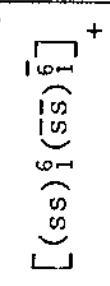 \\
\hline
\end{tabular}




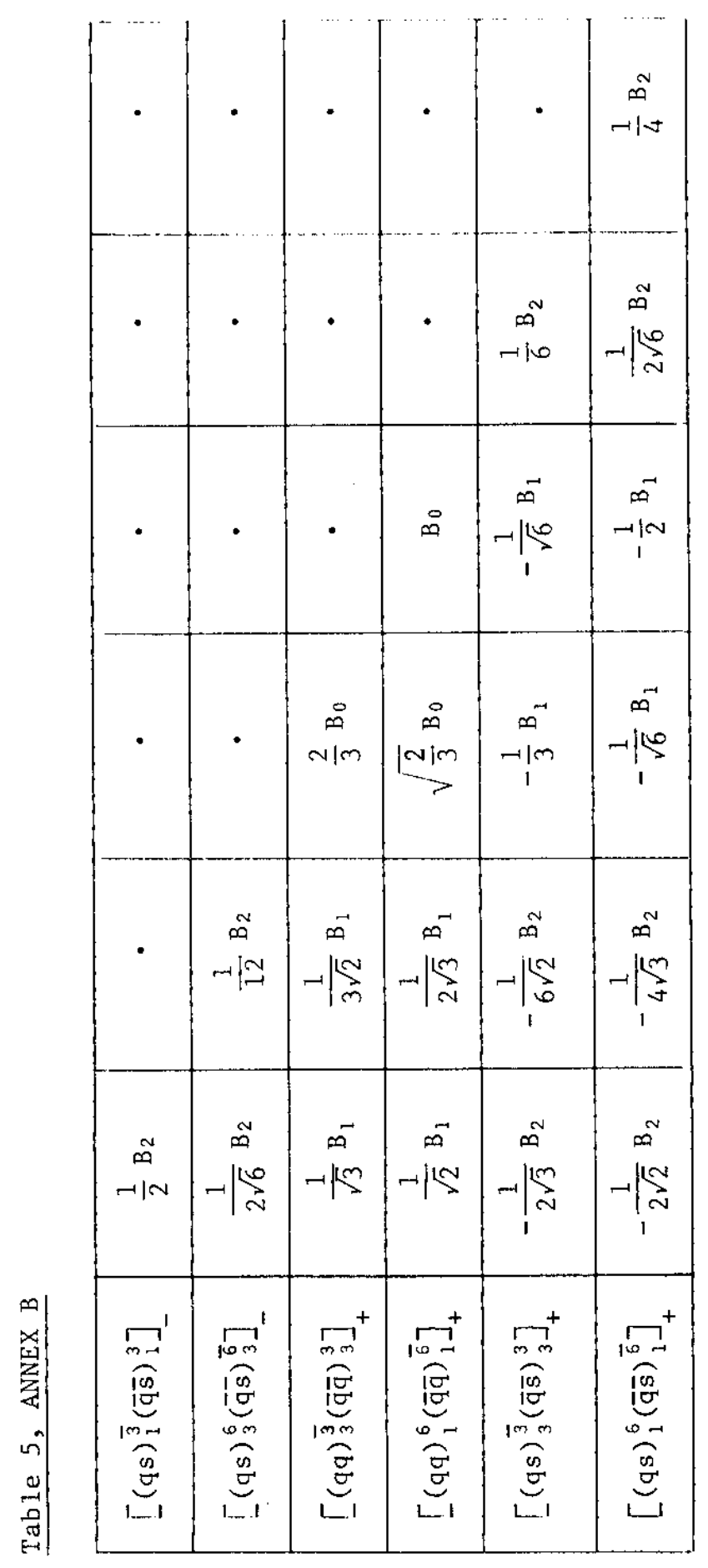


Table 6

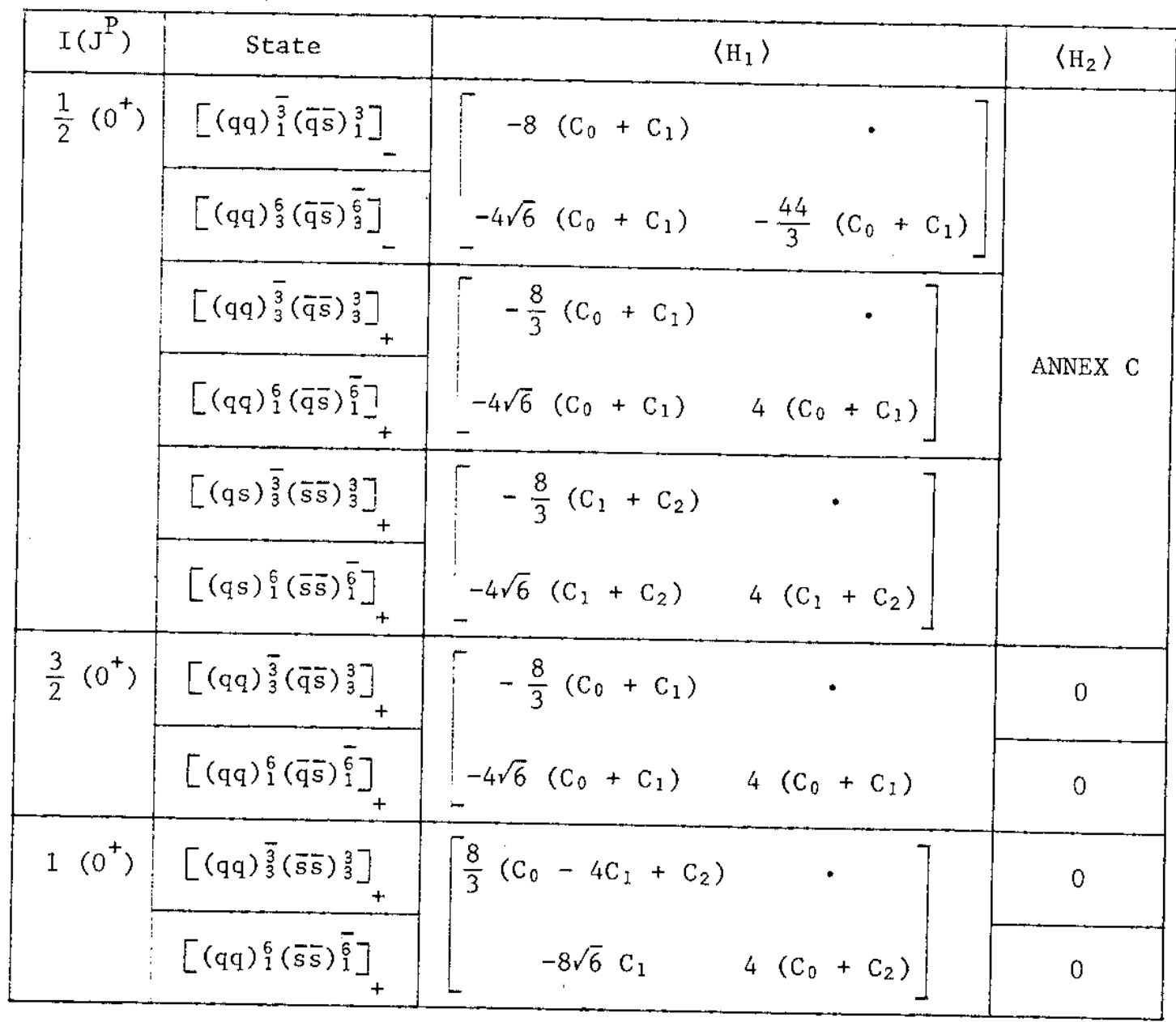

Table 6, ANNEX A

\begin{tabular}{|l|l|l|l|l|l|l|}
\hline$\left[(\mathrm{qq})_{1}^{\overline{3}}(\overline{\mathrm{q}} \overline{\mathrm{s}})_{1}^{3}\right]$ & $\frac{1}{2} \mathrm{~B}_{0}$ & $\cdot$ & $\cdot$ & $\cdot$ & $\cdot$ & $\cdot$ \\
\hline$\left[(\mathrm{qq})_{3}^{6}(\overline{\mathrm{q}} \overline{\mathrm{s}})_{3}^{\overline{6}}\right]_{-}$ & $\frac{1}{2 \sqrt{6}} \mathrm{~B}_{0}$ & $\frac{1}{12} \mathrm{~B}_{0}$ & $\cdot$ & $\cdot$ & $\cdot$ & $\cdot$ \\
\hline$\left[(\mathrm{qq})_{3}^{\overline{3}}(\overline{\mathrm{q}} \overline{\mathrm{s}})_{3}^{3}\right]_{+}$ & $-\frac{1}{2} \mathrm{~B}_{0}$ & $-\frac{1}{2 \sqrt{6}} \mathrm{~B}_{0}$ & $\frac{1}{2} \mathrm{~B}_{0}$ & $\cdot$ & $\cdot$ & $\cdot$ \\
\hline$\left.\left[(\mathrm{qq})_{1}^{6}(\overline{\mathrm{q}} \overline{\mathrm{s}})_{1}^{\overline{6}}\right]_{+}\right]$ & $-\sqrt{\frac{3}{8}} \mathrm{~B}_{0}$ & $-\frac{1}{4} \mathrm{~B}_{0}$ & $\sqrt{\frac{3}{8}} \mathrm{~B}_{0}$ & $\frac{3}{4} \mathrm{~B}_{0}$ & $\cdot$ & $\cdot$ \\
\hline$\left.\left[(\mathrm{qs})_{3}^{\overline{3}}(\overline{\mathrm{s}} \overline{\mathrm{s}})_{3}^{3}\right]_{+}\right]$ & $-\frac{1}{\sqrt{6}} \mathrm{~B}_{1}$ & $-\frac{1}{6} \mathrm{~B}_{1}$ & $\frac{1}{\sqrt{6}} \mathrm{~B}_{1}$ & $\frac{1}{2} \mathrm{~B}_{1}$ & $\frac{1}{3} \mathrm{~B}_{2}$ & $\cdot$ \\
\hline$\left.\left[(\mathrm{qs})_{1}^{6}(\overline{\mathrm{s}} \overline{\mathrm{s}})_{1}^{\overline{6}}\right]_{+}\right]$ & $-\frac{1}{2} \mathrm{~B}_{1}$ & $-\frac{1}{2 \sqrt{6}} \mathrm{~B}_{1}$ & $\frac{1}{2} \mathrm{~B}_{1}$ & $\sqrt{\frac{3}{8}} \mathrm{~B}_{1}$ & $\frac{1}{\sqrt{6}} \mathrm{~B}_{2}$ & $\frac{1}{2} \mathrm{~B}_{2}$ \\
\hline
\end{tabular}


Table 7

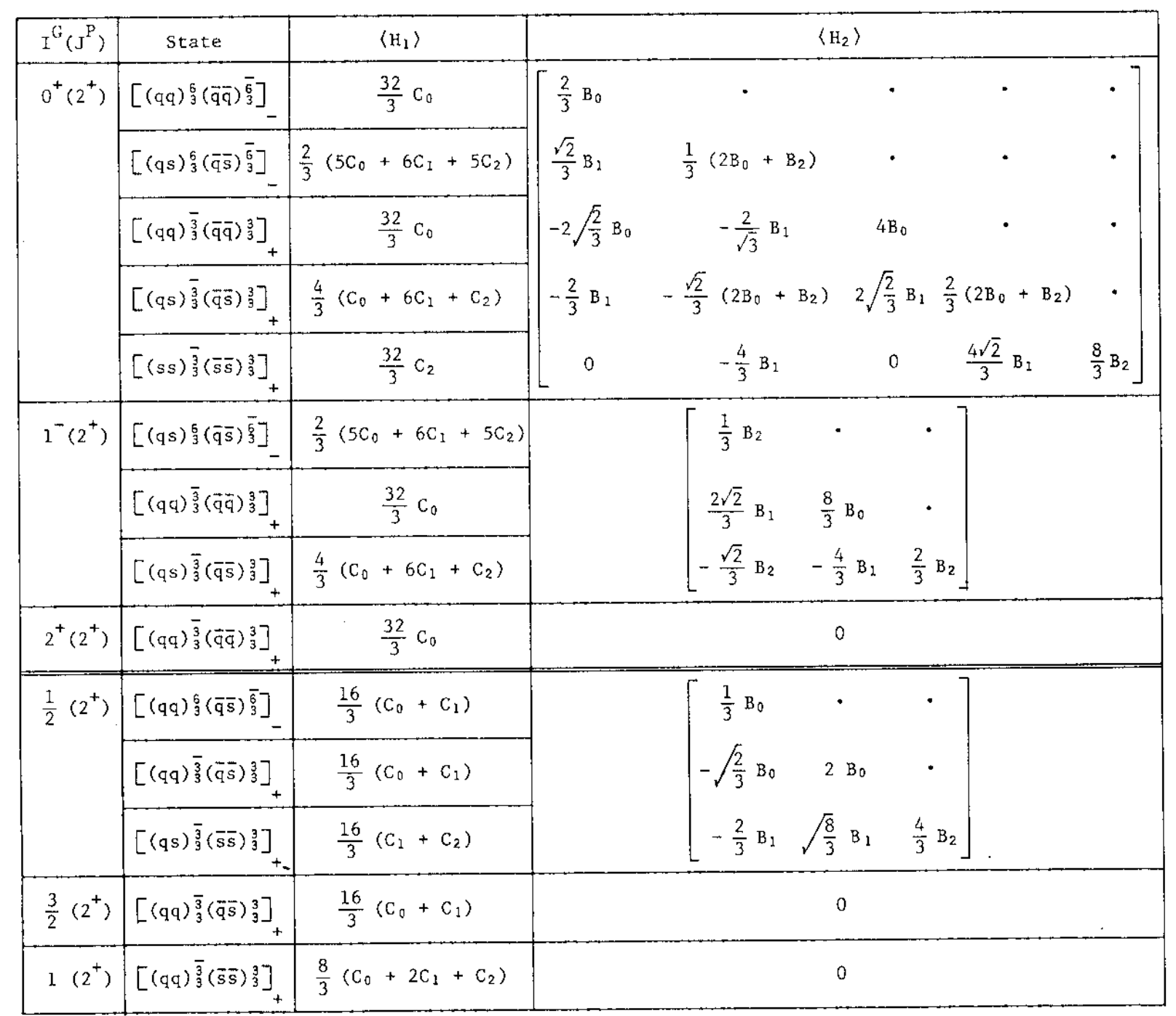



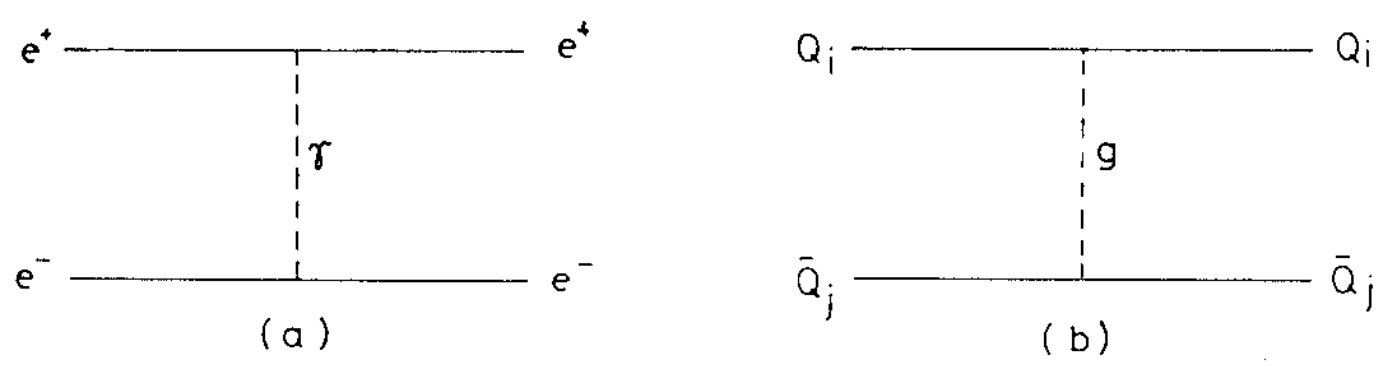

Fig. I
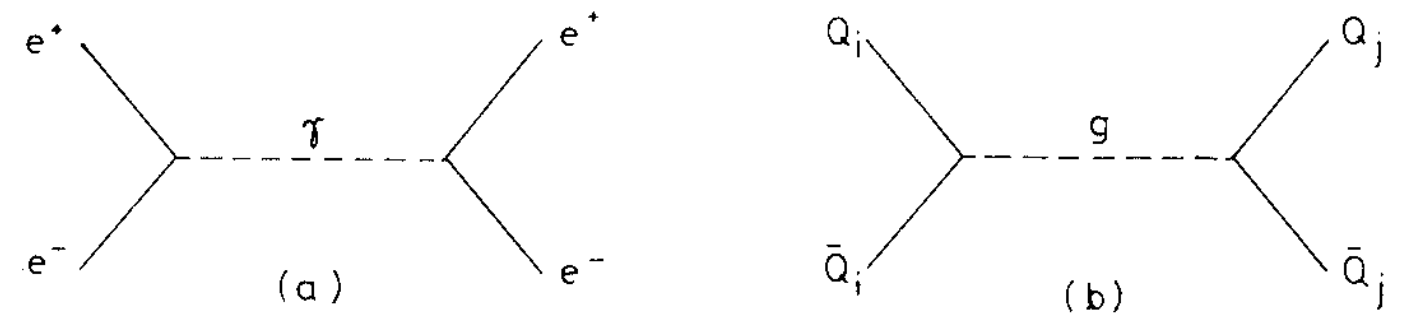

Fig. 2 